\title{
Research Progress on Aluminum-based Composites and Applications
}

\author{
Jinxin $\mathrm{Hu}^{1}$ \\ ${ }^{1}$ South China University of Technology, Guangzhou, 510641, China
}

\begin{abstract}
People propose higher and higher demands for lightweight materials due to the increasing shortage of metals and energy sources. Since traditional aluminum alloy materials cannot meet demands any more, aluminum-based composites attracted high research attentions in recent years because of the excellent mechanical properties and low density. Silicon carbide (SiC) reinforced aluminum-based composite and carbon nanomaterial reinforced aluminum-based composite are two aluminum-based composites which are used mostly in existing associated studies. The former one has a long research history and it has been applied to aerospace, war industry and automobile field to some extent. The later one is an emerging material that is hardly applied yet. This study is going to introduce research progresses and applications of SiC reinforced aluminum-based composite and carbon nanomaterial reinforced aluminum-based composite.
\end{abstract}

\section{Introduction}

Since the development of fused salt electrolysis process in 1866, Al enters into the stage of industrialized mass production. The Al output in China has increased from 7.866 million tons in 2005 to 35.93 million tons in 2019. The development of modern society advocates green economy, energy conservation and emission reduction, which propose many new requirements on materials, including low density, high strength, high rigidity, high tenacity, and adaptation to very tough environments such as strong corrosion, high temperature or ultra-low temperature and high radiation. Although Al alloy material has an application history of more than 100 years, it still has limitations as a metal. On contrast, Al-based composites are superior for the higher strength, higher rigidity, stronger friction resistance and low expansion rate in addition to some excellent properties of Al alloy. This study mainly introduces research progresses and applications of the current mainstream fibers or whisker reinforced Al-based composites as well as the particle reinforced Al-based composites.

\section{Research progresses and applications}

\subsection{SiC particle reinforced Al-based composites}

As by-product from industrial production, $\mathrm{SiC}$ particles incur significantly lower manufacturing costs compared to silicon carbide fiber (SiCF), whisker, monofilament, etc. Moreover, SiCp /Al can also maintain good mechanical properties under unidirectional stress and present isotropic mechanical properties under bidirectional or threedimensional stresses. With the increase of SiC particle content, rigidity, wearing resistance and tensile strength of $\mathrm{SiCp} / \mathrm{Al}$ material are improved significantly, while plasticity declines evidently. Currently, powder metallurgy, squeeze casting, high-energy ultrasonic semi-solid composite technology [1], microwave sintering and heat extrusion technology [2] are common methods to prepare $\mathrm{SiCp} / \mathrm{Al}$ material. However, these methods all face a problem of interface energy between $\mathrm{SiC}$ particles and $\mathrm{Al}$ base. Xu Jincheng et al. [3] found that copper plating on $\mathrm{SiC}$ particles could improve bonding energy of interface effectively, thus enabling to strengthen comprehensive mechanical properties of materials. Dong Cuige et al. [4] coated Titanium onto SiC particles, which improved uniformity and density of composites significantly and increased mechanical performances, while maintaining certain plasticity. Some foreign researchers [5] also applied ultrasonic wave technology to strengthen binding between $\mathrm{SiC}$ particles and $\mathrm{Al}$ base to further increase volume fraction of $\mathrm{SiC}$ particles. At present [6], $\mathrm{SiCp} / \mathrm{Al}$ is used to skin of airplane in addition to engine piston and brake disc in the automobile field. Due to the low expansion rate and good shaping performance, $\mathrm{SiCp} / \mathrm{Al}$ is also extensively applied to tube of satellite T/R component or high-power carrier structure [7]. In recent years, $\mathrm{SiC}$ particles can be used as a kind of functional material by mixing other elements. Elkady et al. [8] pointed out that that the composite which was formed by adding $\mathrm{SiC}$ particles coated with nano $\mathrm{Ni}$ particles and $\mathrm{Ni}$ powder into the Al-based metals could adsorb electromagnetic wave and it could be used as a microwave absorber to replace Iron-based or carbon-based materials of the same type. Since hardness of $\mathrm{SiC}$ is extremely large and $\mathrm{Al}$ alloy is relatively soft, surface quality of materials during precise processing is relatively low. If it uses hard alloy cutter, the cutter may suffer fast abrasion. If ceramic cutter, SCD or 
PCD cutter is applied, blades are easy to fall off due to the long-term alternating loads. Moreover, carbon elements can accelerate wearing of the diamond cutter [9]. At present, some studies chose zone laser melting technology to process parts. The zone laser melting technology is mainly applied to aerospace field and it can not only lower R\&D cost and period of parts effectively, but also achieve relatively good surface quality [10].

\subsection{SiC whisker reinforced Al-based composite}

Compared with SiC particle reinforced Al-based composite, SiC whisker reinforced Al-based composite has better flexural behaviors, tensile performances, elasticity modulus and thermal conductivity. Yue Shanmi et al. [11] prepared SiC whisker on the carbon base by using hydrogen silicon oil as the raw material, which lowered the preparation cost of whisker and increased safety. Nowadays, SiC whisker reinforced Al-based composite attracts increasing attentions from the public, but the $\mathrm{SiC}$ whisker often is mixed into Al-based materials as reinforcement materials together with other ceramic materials, such as aluminum oxide, graphene and carbon nanotubes. Currently, there are single means to prepare $\mathrm{SiC}$ reinforced $\mathrm{Al}$-based composites, mainly using powder metallurgic method. Due to the relatively low Van Der Waals' (VDW) force among whiskers [12-13], SiC whiskers can scatter better in Al-based materials than carbon nanotubes and thereby gain better mechanical properties. As the same with $\mathrm{SiC}$ particle reinforced $\mathrm{Al}$ based composite, $\mathrm{SiC}$ whisker reinforced materials are difficult to be processed. Geng Lin et al. gained workpieces with good surface quality by using diamond cutters and special technological parameters. [14] whisker reinforced Al-based composites which are prepared through casting and powder metallurgic technology often have pores, thus declining mechanical properties (e.g. fatigue strength) of materials. The incremental constraint multidirectional forging method [15] proposed by $\mathrm{Xu}$ Wencheng et al. avoided cracking problem in the forging process, forged pores, loose structures and other defects effectively, and refined crystals to achieve uniform composition and improve mechanical properties of the material. Therefore, the processed materials could be used to manufacture key bearing parts in aerospace or weapon vehicles.

\subsection{Carbon nanotube reinforced Al-based materials}

Carbon nanotube (CNT) is divided into multiwalled carbon nanotube and single-walled carbon nanotube. The later one is hardly studied due to difficulties in preparation and insignificant reinforcement of Al-based materials [16]. CNT reinforced Al-based composite is the nanocarbon reinforced Al-based composite which is studied firstly. Nowadays, powder metallurgic method is the main method to prepare CNT reinforced Al-based composite. CNTs are easy to form clusters because of VDW force, thus resulting in uneven composition of the base and declined properties of materials. In the recent decades, several technologies have been developed, such as highenergy ball milling, flake powder metallurgy, polymer pyrolytic chemical vapor deposition, slurry blending, stirring friction processing. These technologies are beneficial for uniform scattering of CNTs in Al base.

For instance, Wang Lei et al. prepared a composite with uniform distribution of CNTs by assuring uniform growth of CNTs on flake $\mathrm{Al}$ alloy powder by using the chemical vapor deposition (CVD) technology and pressing sintering. The elasticity modulus and tensile strength of the $3 \% \mathrm{CNT} / \mathrm{Al}$ composite were increased by $74.1 \%$ and $31.4 \%$, while the elongation at break declined to $1.5 \%$, which was significantly lower than the base material. [17] Existing studies on CNT reinforced Albased composites mainly focus on how to improve plasticity of materials. After preparing 2\% (mass fraction) CNT/Al through the fake powder metallurgic method, N.Y.Li et al. compacted materials through heat extrusion with high extrusion ratio. CNT and Al base reacted to form Al4C3 to reinforce interface bonding between CNT and Al base. Moreover, CNT generated bridge joint along the extrusion direction, which increased tensile strength of the materials while maintaining plasticity. [18] Finally, elongation of materials was 30\%, accompanied with equivalent plasticity of base material. However, quantity and distribution of Al4C3 are difficult to be controlled. Some researchers implemented electroplating or chemical deposition of silver [19], copper [20] or Ni [21] on CNT surfaces to reinforce bonding between base material and CNT, thus enabling to transfer stress from base materials to reinforcement materials. However, none of them are easy to get uniform and complete cladding layer. X.Q.Liu[22] formed CNTs with TiC through sintering and used it to prepare CNT reinforced Al-based composite. Ti which is a buffer medium forms the TiC-TiAl3-Al structure at the interface, which strengthened the bonding between base and CNTs and increased plasticity and strength of materials. Moreover, smaller size of $\mathrm{Ti}$ particles is more beneficial for load transmission efficiency among interface layers [23]. Electric and thermal conductivities of CNT reinforced Al-based materials are also research hotspots. High-strength $\mathrm{Al}$ conductor is in favor of long-distance power transmission. High-strength Al-based materials can consider electric conductivity while manufacturing structural components, such as some parts in new energy vehicles. At present, agglomeration of CNTs, interface bonding with bases and anistropism of arrangement are main factors that influence thermal and electric conductivities of CNT reinforced Albased materials [24-25]. Chen Liang et al. proposed a preparation technique for sandwich-shaped CNT reinforced Al-based composite, which improved both strength and electric conductivity of materials [26].

\subsection{Graphene reinforced Al-based composite}

Graphene is one of the most potential materials in the 21st Century and there are many preparation methods, including liquid-phase stripping method, redox process, CVD and epitaxial growth method [27]. At present, power metallurgic method, stirring casting method, in-situ 
growth method, pressure penetration method and 3D printing technology [28] are applied to prepare graphene reinforced Al-based composite. The micro-nano laminated powder metallurgic technology [29-30] which was developed by Zhang Di and Li Zhiqiang has built up a production line with annual output of 20 tons. In future, existing $\mathrm{Al}$ alloy material could be replaced to decrease weights by $10 \% \sim 30 \%$. Compared with CNT reinforced Al-based composite, graphene reinforced Al-based composite shows better tensile strength, plasticity and heat and electric conductivities [31-32]. Similar with CNTs, dispersion degree of graphene in base materials and interface bonding with base material are main factors that influence mechanical properties and heat and electric conductivities of graphene Al-based composite. At present, graphene and base metal powder are mixed evenly through ball milling. although ball milling is easy to damage graphene structures, it is the most economic and the simplest method and becomes the first choice of people. The research team of Han Tielong modified graphene surface with low-temperature ball milling technology [33] and nano metallic powder[34], and found that ball milling after coating copper or Ni nano powder on graphene layer can increase dispersion degree of graphene and improve interface bonding effectively compared to direct ball milling under room temperature. MahmoodKhan performed in-situ synthesis of titanium carbide coating on the graphene surface [35], which was cleaner and easier to be controlled than direct coating of a metal layer on graphene surface. Moreover, the interface strength between graphene and the base was improved accordingly. In addition to be a structural component, graphene reinforced Al-based composite can develop new functions by mixing with other elements, such as preparation of antibacterial materials [36] and hydrogen manufacturing material [37].

\section{Conclusions}

The fast development of modern industry proposes higher requirements on materials. Industrial development can break bottlenecks as long as breakthroughs in material performance are achieved. In addition to SiC reinforced Al-based composite, ceramic particle reinforced Al-based composite also includes basalt reinforced Al-based composite [38-39]. Some researchers added CNTs or graphene and ceramic materials together into Al-based composite [40]. Al-based composite materials have to overcome following problems to realize large-scaled applications: 1. Preparation of raw materials, especially, mass production of graphene, CNTs and other carbon fibers. 2) Preparation of composite, including synthesis of metal sheets with stable mechanical properties. 3) Processing of finished products. Existing studies on Albased materials mainly focus on synthesis of materials, and only few studies discussed processing techniques of finished products. Applications of Al-based composite mainly concentrate in aerospace and war industry. The high processing cost restricts Al-based composite within the civil field. With continuous progresses in $\mathrm{R} \& \mathrm{D}, \mathrm{Al}-$ based composite enjoys a promising application prospects and it is beneficial to lower consumptions of metals and energy resources.

\section{References}

1. Yanhua Zhou. (2017) Main preparation technologies for SiC particle reinforced Al-based composite . Tool Engineering, 51(4):7-10.

2. M. Penchal Reddy, R.A. Shakoor, Gururaj Parande, VyasarajManakari, F. Ubaid, A.M.A. Mohamed, Manoj Gupta. (2017) Enhanced performance of nano-sized $\mathrm{SiC}$ reinforced $\mathrm{Al}$ metal matrix nanocomposites synthesized through microwave sintering and hot extrusion techniques, Progress in Natural Science: Materials International, Volume 27, Issue 5, 606-614.

3. Jincheng $\mathrm{Xu}$, Xiaoyan Deng, Chengliang Zhang et al. (2009) Effects of interface improvement of SiC reinforced Al-based composite material on mechanical properties. Material Review, 23 (1); 24-26.

4. Siyun Zhou, Xianliang Zhou, Xia Cui et al. (2017) Effects of chemical copper coating on SiCp surface on corrosion resistance of $\mathrm{SiCp} / \mathrm{Al}$ composite. Journal of Material Heat Treatment, 38(5):6-12.

5. Cui-ge DONG, Rui CUI, Ri-chu WANG, Chaoqun PENG, Zhi-yong CAI, (2020) Microstructures and mechanical properties of Al 2519 matrix composites reinforced with Ti-coated $\mathrm{SiC}$ particles, Transactions of Nonferrous Metals Society of China, Volume 30, Issue 4, Pages 863871,

6. Lin Ma, Changzhuang Zhou, Qi Wen, Mingshen Li, Heng Zhong, Shude Ji, (2020) Ultrasonicpromoted rapid transient liquid phase bonding of high volume fraction $\mathrm{SiC}$ particle reinforced aluminum-based metal matrix composite in low temperature, Ultrasonics, Volume 106, 106159.

7. Beijing Satellite Manufacturing Plant Co., Ltd. Coating of high volume fraction SiC particle reinforced Al-based composites and preparation method: CN201910441172.5[P].2019-09-27.

8. Omayma A. Elkady, Shimaa A. Abolkassem, Ayman H. Elsayed, Walaa A. Hussein, Khalid F.A. Hussein, (2019) Microwave absorbing efficiency of Al matrix composite reinforced with nano$\mathrm{Ni} / \mathrm{SiC}$ particles, Results in Physics, Volume 12, Pages 687-700.

9. Shiyan Huo, Lijing Xie, Junfeng Xiang et al. (2018) Research progresses on ultraprecision machining of $\mathrm{SiC}$ and $\mathrm{SiC}$ particle reinforced $\mathrm{Al}-$ based composites [J]. Tool Engineering, 52(10):312.

10. Yan Zhou, Dongyun Zhang, Weidong Wang et al. (2018) Research status of SiC particle reinforced Al-based composites formed by zone laser melting and applications in aerospace field[J]. Aeronautical Manufacturing Technology, 61(10): 68-73. 
11. Yueshan Mi, Yang Chen, Zhanshen Zheng, Ning Qiao, Yuqi Liang. (2020) New discoveries in the growth of $\mathrm{SiC}$ whiskers derived from hydrogen silicone oil. Journal of Crystal Growth, Volume 543. 125711

12. L.Shahrdami, A. Sedghi, M.H. Shaeri, (2019) Microstructure and mechanical properties of $\mathrm{Al}$ matrix nanocomposites reinforced by different amounts of CNT and SiCW, Composites Part B: Engineering, Volume 175, 07081.

13. Yangyang Wang, Chen Jia, Xian Jia, Jianfeng Yang. (2019) Research status on $\mathrm{SiC}$ whisker reinforced composites [J]. Ordnance Material Science and Engineering, 42(04):142-146.

14. Harbin Institute of Technology. A milling technology for $\mathrm{SiC}$ whisker reinforced Al-based composite: CN201811535088.1[P].2019-04-26.

15. Harbin Institute of Technology. An incremental constraint multidirectional forging method for $\mathrm{SiC}$ whisker reinforced Al-based composite: CN201910839573.6[P].2019-11-29.

16. Yan Shi, Lei Zhao, Zan Li, Zhiqiang Li, DingBang Xiong, Yishi Su, Shmuel Osovski, QiangGuo, (2019) Strengthening and deformation mechanisms in nanolaminated single-walled carbon nanotube-aluminum composites, Materials Science and Engineering: A, Volume 764, 138273

17. Wang Lei, Yin Hua, Xu Run et al. (2019) Preparation of In-situ CNT/Al-based composite and mechanical properties. Materials Science and Engineering of Powder Metallurgy Materials, 24(1):63-67, 74.

18. N.Y. Li, C. Yang, C.J. Li, H.D. Guan, D. Fang, J.M. Tao, Y.C. Liu, J.H. Yi, (2020) Carbon nanotubes reinforced aluminum matrix composites with high elongation prepared by flake powder metallurgy, Diamond and Related Materials, Volume 107, 107907.

19. Qi Zhao, Ming Xie, Yichun Liu, Jianhong Yi, (2017) Improved electroless plating method through ultrasonic spray atomization for depositing silver nanoparticles on multi-walled carbon nanotubes, Applied Surface Science, Volume 409, Pages 164-168.

20. M.Jagannatham, S. Sankaran, Prathap Haridoss, (2015) Microstructure and mechanical behavior of copper coated multiwall carbon nanotubes reinforced aluminum composites, Materials Science and Engineering: A, Volume 638, Pages 197-207.

21. O. Carvalho, M. Buciumeanu, S. Madeira, G. Miranda, F.S. Silva, (2016) Interface analysis on an eutectic AlSi alloy reinforced with $\mathrm{Ni}$ coated MWCNT, Composites Part B: Engineering, Volume 93, Pages 229-235.

22. X.Q. Liu, C.J. Li, J.H. Yi, K.G. Prashanth, N. Chawake, J.M. Tao, X. You, Y.C. Liu, J. Eckert, (2018) Enhancing the interface bonding in carbon nanotubes reinforced $\mathrm{Al}$ matrix composites by the in situ formation of $\mathrm{TiAl} 3$ and $\mathrm{TiC}$, Journal of Alloys and Compounds, Volume 765, Pages 98105.

23. X.Q. Liu, C.J. Li, X. You, Z.Y. Xu, X. Li, R. Bao, J.M. Tao, J.H. Yi, (2020) Size-dependent effects of $\mathrm{Ti}$ powders in the pure aluminum matrix composites reinforced by carbon nanotubes, Journal of Alloys and Compounds, Volume 823, 153824.

24. Mengwu Wu, Lin Hua, Jianxin Zhou et al. (2018) Research progresses of heat conducting Al alloy and Al-based composite . Materials Review, 32(9):1486-1495.

25. Rui Sun, Yi Qing, Jingwei Zhuang et al. (2019) Research progress on electric conductivity of $\mathrm{Al}$ alloy and Al-based composite [J]. Light Alloy Fabrication Technology, 47(2):9-15, 37.

26. Shandong University. A CNT reinforced multilayer Al-based composite and its preparation method and applications. CN201910614823.6[P].2019-10-11.

27. Jiangyu Li, Shourong Zhao, Wei Zhang et al. (2019) Research progresses on graphene reinforced Al-based composite . Material Science, 9(08):803-812.

28. Denghao Yi, Yinghao Feng, Jinfang Zhang et al. (2020) Research progresses on 3D printed graphene reinforced composite . Material Review, 34(9):9086-9094.

29. Bozhang Qian, (2019) Graphene becomes the "trump card" of aluminum alloy and increases traveling speed of big airplanes and high-speed trains made in China [J]. Synthetic Materials Aging and Application, 48(6):141.

30. Zhiqiang Li. (2015) Combined preparation technology of Al-based nano alloy and flake powder metallurgy / bionics [C]. // Chinese Materials Research Society. Memoir for the 15th National Scientific and Technological Seminar of Youth Materials of China, the 6th Scientific and Technological Seminar for Youth Materials in China and Foreign Countries. Chongqing, 109109.

31. Shuai Zhang, Gaoqiang Chen, Timing Qu, Gang Fang, Shengwen Bai, Yufan Yan, Gong Zhang, Zhaoxia Zhou, Junjun Shen, Dawei Yao, Yuanwang Zhang, QingyuShi, (2020) Simultaneously enhancing mechanical properties and electrical conductivity of aluminum by using graphene as the reinforcement, Materials Letters, Volume 265, 127440

32. Alicia Weibel, Andréas Flaureau, Adeline Pham, Geoffroy Chevallier, Jérôme Esvan, Claude Estournès, Christophe Laurent, (2020) One-step synthesis of few-layered-graphene/alumina powders for strong and tough composites with high electrical conductivity, Journal of the European Ceramic Society, Volume 40, Issue 15, Pages 5779-5789 
33. Tielong Han, Jiajun Li, Naiqin Zhao, Chunnian He, (2020) Microstructure and properties of copper coated graphene nanoplates reinforced $\mathrm{Al}$ matrix composites developed by low temperature ball milling, Carbon, Volume 159, Pages 311-323

34. Tielong Han, Enzuo Liu, Jiajun Li, Naiqin Zhao, Chunnian He, (2020) A bottom-up strategy toward metal nano-particles modified graphene nanoplates for fabricating aluminum matrix composites and interface study, Journal of Materials Science \&Technology, Volume 46, Pages 21-32.

35. Mahmood Khan, Salman Ahmad, Sarim Zaidi, Abdul Wadood, Tayyab Subhani, Shahid Akhtar, Syed Wilayat Husain, Ragnhild Elizabeth Aune, (2020) Titanium carbide coating on graphene nanoplatelets, Journal of Materials Research and Technology, Volume 9, Issue 3, Pages 3075-3083.

36. Zhipo Zhao, Fanchi Meng, Junrong Tang, Housheng Liu, Hongyang Liu, Lini Yang, Jiqiang Wang, Tianying Xiong, (2019) A novel method of fabricating an antibacterial aluminum-matrix composite coating doped graphene/silvernanoparticles, Materials Letters, Volume 245, Pages 211-214.

37. Fei Xiao, Rongjie Yang, Jianmin Li, (2020) Aluminum composites with bismuth nanoparticles and graphene oxide and their application to hydrogen generation in water, International Journal of Hydrogen Energy, Volume 45, Issue 11, Pages 6082-6089.

38. Caihe Fan, Zeyi Hu, Xihong Chen et al. (2020) Interface structure and mechanical behaviors of basalt particle reinforced 7A04 Al-based composite. Chinese Journal of Nonferrous Metals, 30(1):40-47.

39. Hao Ding, Xiping Cui, Changshou XU et al. (2018) Preparation and mechanical properties of continuous basalt fiber reinforced Al-based laminated composite. Acta Metall Sinica, 54(8):1171-1178.

40. Shenyang Ligong University. Graphene-SiC reinforced Al-based composite and preparation method. CN201811159446.3[P].2019-01-01. 